\title{
A Better Characterization of Brain Damage in Carbon Monoxide Intoxication Assessed in Vivo Using Diffusion Kurtosis Imaging
}

\author{
Wen-Yao FAN ${ }^{1}$, Wen-Bin ZHENG $^{1, a, ~}{ }^{*}$, Xi-Ran CHEN ${ }^{1}$
}

Departments of Radiology, the Second Affiliated Hospital, Medical College of Shantou University, Shantou, China

aemail: hwenb@126.com

${ }^{*}$ Corresponding author: Tel: 86075488915717 , Fax: 86075483141198

Keywords: Carbon monoxide poisoning, Diffusion kurtosis imaging.

\begin{abstract}
Diffusion kurtosis imaging findings in delayed encephalopathy after acute carbon monoxide poisoning have seldom reported. We present a study of a 25 -yearold female with acute carbon monoxide poisoning by using conventional magnetic resonance imaging (MRI) and diffusion kurtosis imaging (DKI). Conventional brain MR imaging on day-6 after Carbon monoxide (CO) poisoning showed hyperintensities in the bilateral temporal lobe, hippocampus and basal ganglia in T2weighted imaging. Compared with the healthy age-matched control group $(n=5)$, in day-16, the values measured in the patient on DKI showed MD values were significantly increased and FA values were decreased in the cerebellum $(p<0.05$, all), MK values were increased in the basal ganglia $(p<0.05)$. On week-20, FA values decreased and MD values increased in the basal ganglia $(p<0.05)$. MK values decreased in the basal ganglia but with no statistical significance. MRI showed only abnormal hyperintensities remained in the basal ganglia on T2WI. As sensitive imaging techniques, DKI can provide a more comprehensive characterization of damage in patients after acute carbon monoxide poisoning, and provide additional and complementary information to DTI on pathophysiology. A higher MK value of the lesion in early stage may strongly suggest the poor prognosis of carbon monoxide poisoning.
\end{abstract}

\section{Introduction}

Acute carbon monoxide poisoning can lead to multi-system organ lesions. Damage to the brain affects the prognosis for patients who survive CO poisoning directly. Delayed encephalopathy after acute carbon monoxide poisoning (DEACMP) is a serious complication of carbon monoxide intoxication. Predicting whether patients who exhibit resolved acute symptoms have escaped or will experience delayed neuropsychiatric sequelae (DNS) represents a very important clinical issue [1].One of the strategies to resolve these clinical problems is to apply objective assessments of brain damage by using MR imaging, a readily available and minimally invasive procedure [2]. Diffusion Kurtosis Imaging (DKI) has been shown to be sensitive to structural changes in both anisotropic tissue, such as WM, and isotropic tissue such as GM and therefore may provide more information than DTI on tissue microarchitecture complementary [3]. Diffusion kurtosis imaging findings in delayed encephalopathy after acute carbon monoxide poisoning have seldom reported. The aim of our study is to investigate: 1) Regions of WM and GM microstructural abnormalities in the patients after acute carbon monoxide poisoning by using DKI. 2) Whether DKI-derived parameter, the mean kurtosis (MK), provides additional 
information about the underlying structural abnormalities after acute carbon monoxide poisoning compared with mean diffusivity (MD) and fractional anisotropy (FA).

\section{Case Report}

A 25-year-old woman who was found lapsed into unconsciousness after using the coal-fired water heater to take a bath, and then she was taken by ambulance to a hospital. Her arterial carboxyhemoglobin level on admission was 52.0\%, and Computed tomography (CT) of her brain showed generalized brain swelling. Based on the clinical history and Laboratory/imaging findings, the diagnosis of carbon monoxide poisoning was assumed. After hospital admission, she was treated with hyperbaric oxygen (HBO) and with intravenous corticosteroids and edaravone, a free radical scavenger. After positive treatments, she gradually recovered consciousness. She underwent magnetic resonance imaging (MRI) on day-6, day-16, week-20 after carbon monoxide poisoning by using a GE 3.0-T MR scanner equipped. One-way ANOVA was used in statistical analysis for comparing changes of DKI values in different areas of the brain.

Conventional brain MR imaging on day-6 after carbon monoxide poisoning showed abnormal hyperintensities in the bilateral temporal lobe, hippocampus and basal ganglia in T2-weighted imaging (Fig. 1). Ten days later, on day-16 of hospitalization, the patient's intelligent and motor impairment symptoms like shortterm memory declined, computed capacity declined, tremor and dystonia was still not improved, but she had a favorable conventional MRI evolution, MR imaging (Fig. 2) showed hippocampus and basal ganglia with normal signal intensity in T2-weighted imaging, only abnormal hyperintensities in the bilateral temporal lobe were observed. On week 20 , the patient complained for some remained symptoms, especially shortterm memory declined. MR imaging (Fig. 3) showed the abnormal signal intensity in bilateral temporal lobe was also disappeared on T2-weighted imaging, but hyperintense in the basal ganglia were observed. Further imaging study was by diffusion kurtosis imaging (DKI) (Fig. 4) showed MD values were increased and FA values or MK values were decreased in the cerebellum $(P<0.05$, all), and only MK values were significant increased in the basal ganglia $(P<0.05)$ on day-16 of hospitalization. On week 20, FA values decreased and MD values increased in the basal ganglia were observed $(P<0.05$, all $)$, FA values decreased in the hippocampus and MD increased in temporal lobe were also founded $(P<0.05$, all). MK values decreased in all the measured areas without statistical significance. (Table 1)

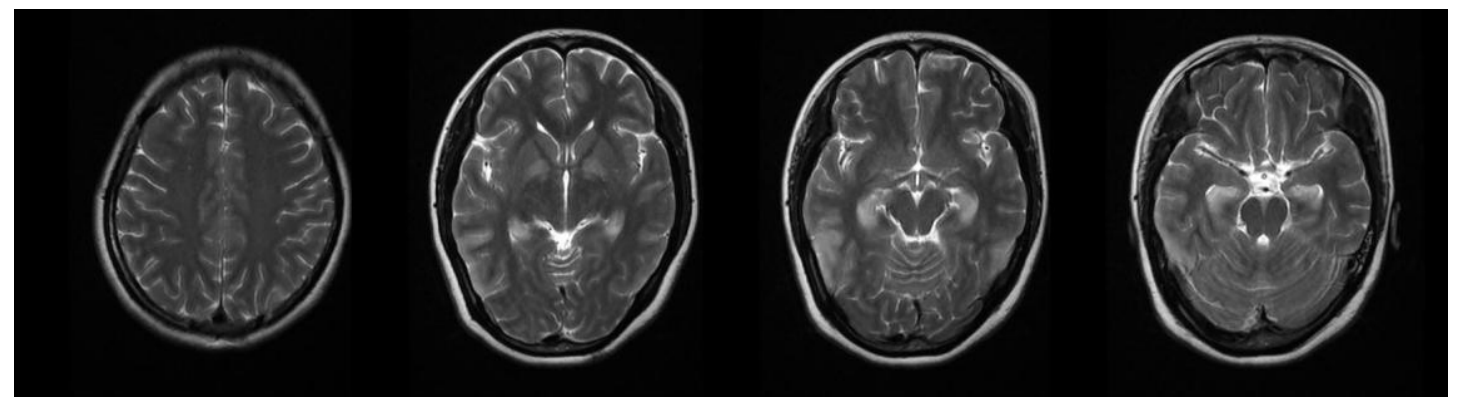

Figure 1. T2-weighted imaging (T2WI) on day-6 of hospitalization showed hyperintensities in the bilateral temporal lobe, hippocampus and basal ganglia. 


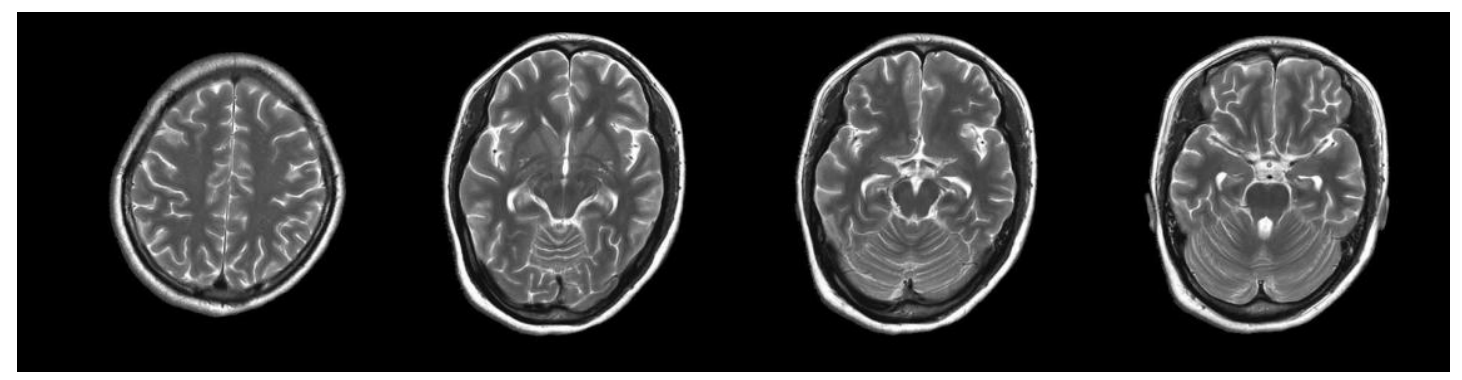

Figure 2. T2-weighted imaging (T2WI) on day-16 of hospitalization showed normal appearing in the hippocampus and basal ganglia and abnormal hyperintensities in the bilateral temporal lobe were significantly reduced.

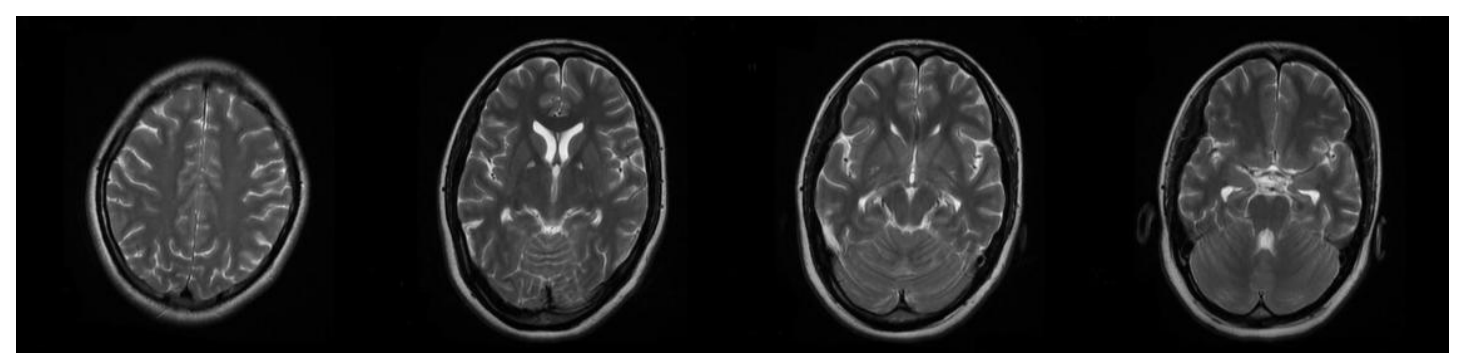

Figure 3. T2-weighted imaging (T2WI) on week-20 after discharging from hospital showed normal appearing in the hippocampus and hyperintense in the basal ganglia.

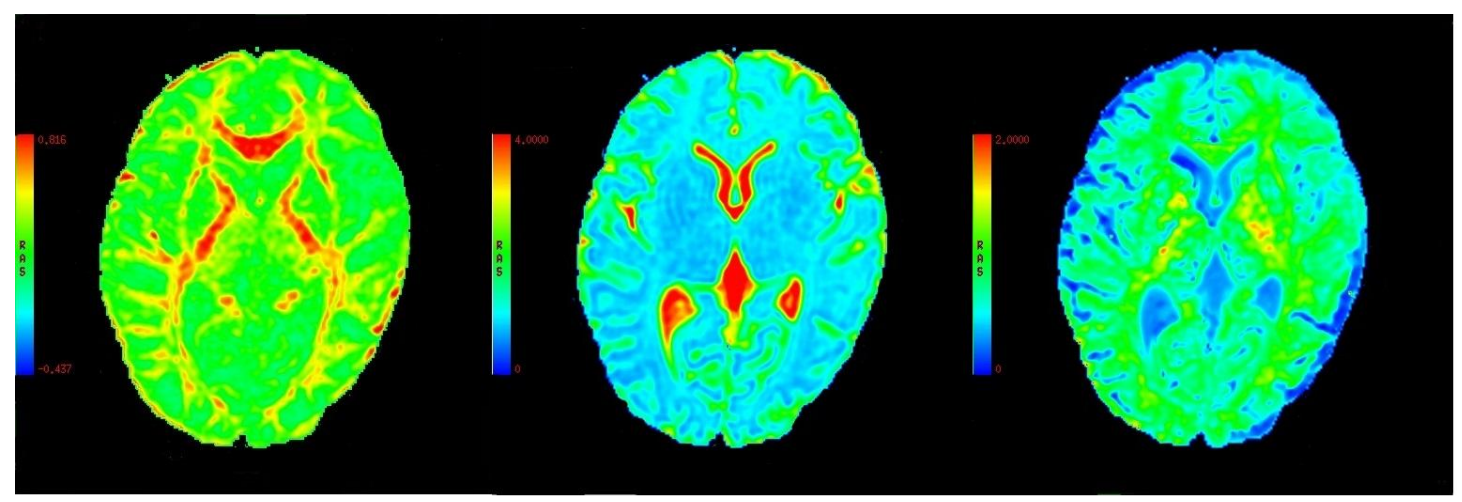

Figure 4. FA, MD and MK maps of the patient on day-16.

Table 1. Diffusion kurtosis imaging derived values based on the patient and corresponding areas in healthy controls. Data are means (of voxels) \pm inter-subject SD

\begin{tabular}{|c|c|c|c|c|c|}
\hline & Brain region & Basal ganglia & Temporal Lobe & Cerebellum & Hippocampu \\
\hline \multirow{4}{*}{ FA } & $\begin{array}{c}\text { Control subjects } \\
(\mathrm{n}=5)\end{array}$ & $0.37 \pm 0.06$ & $0.36 \pm 0.14$ & $0.34 \pm 0.04$ & $0.41 \pm 0.07$ \\
\cline { 2 - 6 } & Patient (day 16) & $0.40 \pm 0.08$ & $0.37 \pm 0.07$ & $0.29 \pm 0.02^{*}$ & $0.38 \pm 0.02$ \\
\cline { 2 - 6 } & Patient (week 20) & $0.29 \pm 0.02^{*}$ & $0.31 \pm 0.03$ & $0.30 \pm 0.02^{*}$ & $0.32 \pm 0.03^{*}$ \\
\hline \multirow{3}{*}{ MD } & $\begin{array}{c}\text { Control subjects } \\
(\mathrm{n}=5)\end{array}$ & $0.79 \pm 0.19$ & $0.76 \pm 0.17$ & $0.66 \pm 0.15$ & $0.79 \pm 0.21$ \\
\cline { 2 - 6 } & Patient (day 16) & $0.84 \pm 0.04$ & $0.89 \pm 0.11$ & $0.96 \pm 0.12^{*}$ & $0.81 \pm 0.10$ \\
\cline { 2 - 6 } & Patient (week 20) & $0.98 \pm 0.03^{*}$ & $0.97 \pm 0.02^{*}$ & $0.83 \pm 0.03$ & $0.95 \pm 0.05$ \\
\hline \multirow{4}{*}{ MK } & $\begin{array}{c}\text { Control subjects } \\
(\mathrm{n}=5)\end{array}$ & $0.87 \pm 0.14$ & $0.87 \pm 0.28$ & $1.07 \pm 0.21$ & $0.78 \pm 0.14$ \\
\cline { 2 - 6 } & Patient (day 16) & $1.12 \pm 0.11^{*}$ & $0.79 \pm 0.05$ & $0.82 \pm 0.21^{*}$ & $0.81 \pm 0.10$ \\
\cline { 2 - 6 } & Patient (week 20) & $0.66 \pm 0.11$ & $0.79 \pm 0.09$ & $0.91 \pm 0.05$ & $0.68 \pm 0.05$ \\
\hline
\end{tabular}

* $\mathrm{P}<0.05$ compared with controls 


\section{Discussion}

As mentioned above, pathologic changes induced by complicated mechanisms progress in the brain from the time of $\mathrm{CO}$ inhalation to even some years later. Most articles described findings in the chronic phase and documented typical findings of bilateral hyperintensities in the GP and CWM on T2-weighted images $[4,5]$. In these reports, the authors speculated that the main pathologic changes depicted on MR imaging were repairable demyelination in the cerebellum, and necrosis in the basal ganglia, which leaded to delayed encephalopathy.

DTI, which can show the directionality of water molecule diffusion, reflect the pathologic changes of microstructural in brain tissue which conventional MRI cannot. Diffuse characteristics of DTI (ADC, FA) can accurately reflect different pathological changes of white matter microstructure, and quantify the degree of brain impairment. The FA value reflects the direction of the water molecular diffusion, mainly related to white matter fiber tissue density, and the number of myelin damage, provides information about the cell arrangement and the structural integrity of white matter tracts $[6,7]$. These phenomena might indicate demyelination of myelinated CWM. DTI has been applied to evaluate CO poisoning since approximately 2005 [8-11]. However the DTI model is based on the assumption that the displacement probability function of diffusing water molecules follows a Gaussian distribution, which is only the first approximation to the true behavior in complex tissue. Therefore, while DTI can be used to measure the diffusional motion of water molecules, and can provide a unique source of contrast among white matter structures, but provides limited diagnostic value of grey matter structure.

To assess brain damage (WM and GM) secondary to CO poisoning, we use a new sequence, diffusional kurtosis imaging (DKI), allowing more comprehensive characterization of microstructural changes in the brain $[12,13,14]$. We investigated $\mathrm{MK}$, as a DKI quantitative value, not only in abnormal signal regions but also in normal appearing region, and the findings were remarkable.

MRI study showed the patient were with obviously abnormal hyperintensities on T2WI in temporal lobe, hippocampus and basal ganglia on acute stage after carbon monoxide poisoning, and on subacute to chronic stage, some of the lesions disappeared and some of them remained. It suggested that angioedema as a result of acute cerebral hypoxia in the bilateral temporal lobe and hippocampus might be temporary, which could recover completely without residual disability. On the other hand, compared with the control group, DKI examination revealed only a high MK value of basal ganglia in the subacute stage, and in the chronic stage,we also found only abnormal hyperintensities remained in the basal ganglia on T2WI. Previous DKI studies about cerebral infarction had showed that MK value of the acute phase of cerebral ischemia was significantly higher than that of normal tissue [15], which indicated that increased MK values in the initial stages can be an early prognostic factor of poor prognosis. So we believe increased MK values in early stage of carbon monoxide intoxication might correlate to poor prognosis.

Although the cerebellum was with normal appearing on T2WI, FA and MK value of the cerebellum were decreased and MD value increased on day-16, and there was a statistically significantly difference between patient and the control group. On week 20, there was no difference in MK values of cerebellum between patient and the control group, and the patient's motor impairment symptoms like tremor and dystonia were no longer existed. So lesions with decreased of FA and MK value and increased of MD in early stage were consistent with the pathological changes of acute cerebral 
edema, capillary and venous dilatation induced by cerebral hypoxia and might not correlated to the prognosis of carbon monoxide intoxication.

In conclusion, as sensitive imaging techniques, DKI combining conventional MRI can be valuable to probe the injury of brain micro structures, it can provide a more comprehensive characterization of lesions and normal-appearing WM and gray matter damage in patients after acute carbon monoxide poisoning. A higher MK value of the lesion in early stage may strongly suggesting the A higher MK value of the lesion in early stage may strongly suggesting the poor prognosis of carbon monoxide poisoning.

\section{Acknowledgments}

This study was supported by the National Natural Science Foundation of China (grant No.81571627), Natural Science Foundation of Guangdong Province, China (grant No. 2014A030313481), and was sponsored by Shantou University Innovation project, Shantou China, Science and Technology Planning Project of Shantou City, China (grant No.201424260), Shantou China. WB Zheng received the Funding.

\section{References}

[1] Beppu T, Nishimoto H, Ishigaki D, et al. Assessment of damage to cerebral white matter fiber in the subacute phase after carbon monoxide poisoning using fractional anisotropy in diffusion tensor imaging. Neuroradiology 2010; 52: 735-43

[2] Fieremans E, Benitez A, Jensen $\mathbf{J ~ H}$, et al. Novel white matter tract integrity metrics sensitive to Alzheimer disease progression[J]. American Journal of Neuroradiology, 2013, 34(11): 2105-2112.

[3] Raz, Eytan, et al. "A better characterization of spinal cord damage in multiple sclerosis: a diffusional kurtosis imaging study." American Journal of Neuroradiology 34.9 (2013): 1846-1852.

[4] Horowitz AL, Kaplan R, Sarpel G. Carbon monoxide toxicity: MR imaging in the brain. Radiology 1987; 162: 787-88

[5] Vieregge P, Klostermann W, Blümm RG, et al. Carbon monoxide poisoning: clinical, neurophysiological, and brain imaging observations in acute disease and follow-up. J Neurol1989; 236: 478-81

[6] Kim JH, Chang KH, Song IC, et al. Delayed encephalopathy of acute carbon monoxide intoxication: diffusivity of cerebral white matter lesions. AJNR Am J Neuroradiol 2003; 24: 1592-97

[7] Teksam M, Casey SO, Michel E, et al. Diffusion-weighted MR imaging findings in carbon monoxide poisoning. Neuroradiology 2002; 44: 109-13

[8] Lo CP, Chen SY, Chou MC, et al. Diffusion-tensor MR imaging for evaluation of the efficacy of hyperbaric oxygen therapy in patients with delayed neuropsychiatric syndrome caused by carbon monoxide inhalation. Eur J Neurol 2007;14:777-82.

[9] Vila JF, Meli FJ, Serqueira OE, et al. Diffusion tensor magnetic resonance imaging: a promising technique to characterize and track delayed encephalopathy after acute carbon monoxide poisoning.Undersea Hyperb Med 2005; 32: 151-56; 
[10] Terajima $\mathrm{K}$, Igarashi $\mathrm{H}$, Hirose $\mathrm{M}$, et al.Serial assessments of delayed encephalopathy after carbon monoxide poisoning using magnetic resonance spectroscopy and diffusion tensor imaging on 3.0T system. Eur Neurol 2008;59:5561 .

[11] Beppu, T. "The role of MR imaging in assessment of brain damage from carbon monoxide poisoning: a review of the literature." American Journal of Neuroradiology 35.4 (2014):625-631.

[12] Jensen JH, Helpern JA. MRI quantification of non-Gaussian water diffusion by kurtosis analysis. NMR Biomed 2010; 23: 698-710

[13] Jensen JH, Helpern JA, Ramani A, et al. Diffusional kurtosis imaging: the quantification of non-gaussian water diffusion by means of magnetic resonance imaging. Magn Reson Med 2005; 53: 1432-40

[14] Fieremans, E., et al. "Novel white matter tract integrity metrics sensitive to Alzheimer disease progression." American Journal of Neuroradiology 34.11 (2013): 2105-2112.

[15] Jensen JH, Falangola MF, Hu C, et a1.Preliminary observations of increased diffusional kurtosis in human brain following recent cerebral infarction. NMR Biomed, 2O11, 24(5): 452- 457. 
